\title{
Rapid Detection of Ebola Virus with a Reagent-Free, Point-of-Care Biosensor
}

\author{
Justin T. Baca ${ }^{1, *}$, Virginia Severns ${ }^{2}$, Debbie Lovato ${ }^{2}$, Darren W. Branch ${ }^{3}$ \\ and Richard S. Larson ${ }^{2}$
}

1 Department of Emergency Medicine, University of New Mexico School of Medicine, Albuquerque, NM 87131, USA

2 Department of Pathology, University of New Mexico School of Medicine, Albuquerque, NM 87131, USA; E-Mails: VSeverns@salud.unm.edu (V.S.); DLovato@salud.unm.edu (D.L.); RLarson@salud.unm.edu (R.S.L.)

3 Sandia National Laboratories, Albuquerque, NM 87185, USA; E-Mail: dwbranc@sandia.gov

* Author to whom correspondence should be addressed; E-Mail: JTBaca@salud.unm.edu; Tel.: +1-505-272-5062; Fax: +1-505-272-6503.

Academic Editor: Stephane Evoy

Received: 27 February 2015 / Accepted: 9 April 2015 / Published: 14 April 2015

\begin{abstract}
Surface acoustic wave (SAW) sensors can rapidly detect Ebola antigens at the point-of-care without the need for added reagents, sample processing, or specialized personnel. This preliminary study demonstrates SAW biosensor detection of the Ebola virus in a concentration-dependent manner. The detection limit with this methodology is below the average level of viremia detected on the first day of symptoms by PCR. We observe a log-linear sensor response for highly fragmented Ebola viral particles, with a detection limit corresponding to $1.9 \times 10^{4} \mathrm{PFU} / \mathrm{mL}$ prior to virus inactivation. We predict greatly improved sensitivity for intact, infectious Ebola virus. This point-of-care methodology has the potential to detect Ebola viremia prior to symptom onset, greatly enabling infection control and rapid treatment. This biosensor platform is powered by disposable AA batteries and can be rapidly adapted to detect other emerging diseases in austere conditions.
\end{abstract}

Keywords: pathogens; infectious diseases; viruses; piezoelectric; biosensors; diagnosis; SAW; surface acoustic wave 


\section{Introduction}

The 2014 Ebola virus outbreak is the largest in history, with widespread transmission in multiple West African countries and sporadic cases in Europe and North America [1-4]. The massive public health response has been limited, in part, by the inability to rapidly detect the presence of Ebola virus in potential patients living in remote areas [5]. The early symptoms of Ebola overlap with symptoms of endemic malaria and other febrile illnesses [6].

Rapid, point-of-care detection of the Ebola virus could enable early quarantine and halt future epidemics and pandemics [5,6]. While point-of-care nucleotide amplification tests exist [5], these are limited by the need for multiple reagents, refrigeration, and specialized personnel. Here, we present preliminary results of a Surface Acoustic Wave (SAW) biosensor with potential to detect Ebola virus in multiple, unprocessed sample types including blood, serum, saliva, and feces within 5-10 min.

Ebola virus is a class A select agent filovirus that was first identified in Zaire in 1976 and named after the River Ebola in Zaire [7]. Ebola outbreaks are sustained through person-to-person transmission from direct contact with infected people, bodily fluids, or contaminated clothes and linens [7]. Early detection of this highly contagious virus would allow for improved infection control measures and early treatment at specialized facilities. The current mortality rate from epidemic Ebola ranges from $40 \%$ to $90 \%$ and could be greatly reduced with early, point-of-care diagnostics [8,9]. Mathematical models suggest that the total direct costs of the present outbreak range from $\$ 82$ million to $\$ 356$ million in the three most effected countries; early diagnosis and treatment will also reduce aggregate healthcare costs [10].

Current approaches to Ebola identification include antigen-capture ELISA testing, IgM ELISA, RT-PCR, virus isolation, electron microscopy, and serologic testing for $\operatorname{IgM}$ or $\operatorname{IgG}$ antibodies. Quantitative measurement of the viral load also has prognostic importance, with a much higher case fatality rate in patients with viral loads over 10 million copies per milliliter [11]. Current tests for Ebola, especially PCR-based techniques, are generally limited by the need for added reagents, refrigeration, and the need for specially trained laboratory personnel. Lateral flow assays can provide rapid and fairly inexpensive qualitative results, but are unable to quantify viral load.

Our group has developed a point-of-care biosensor that employs surface acoustic waves for label-free pathogen detection without the need for any sample preparation [12-14]. This biosensor platform detects target antigens in the presence of confounding analytes and in various types of media, thus eliminating complicated sample preparation protocols. Such acoustic wave sensors can achieve limits of detection of $<50 \mathrm{pg} / \mathrm{cm}^{2}$, which is an order of magnitude lower than detection limits for other marker-free systems such as optical surface plasmon resonance (SPR) and quartz crystal microbalance (QCM) devices [15].

With this sensor platform, viral detection is rapid (with acoustic wave signal detection within 2 min), and the entire detection protocol (from collecting the test sample to obtaining a positive or negative test result) requiring about 5-10 $\mathrm{min}$. Such technological benefits would be ideal for rapid, point-of-care identification and diagnosis of the Ebola virus, especially in environments with minimal or overloaded infrastructure, such as in public health or emergency response situations. Here, we discuss initial modifications of the biosensor to demonstrate detection of Ebola and its potential utility to combat this recurrent epidemic. 


\section{Experimental Section}

\subsection{Fabrication and Functionalization of the SAW Biosensor}

The sensor chips were prepared using lithium tantalate $\left(36^{\circ}\right.$, y-cut, x-propagating $\left.\mathrm{LiTaO}_{3}\right)$ wafers by lithographic deposition and patterning of inter-digital transducers (IDT) and waveguide layers as previously described [12]. Briefly, cleaned $\mathrm{LiTaO}_{3}$ wafers were patterned with four IDT patterns with sets of transducers and delay lines using negative tone photoresist AZ2020 (AZ Electronic Materials, Branchburg, NJ, USA). Then, a metallization step was performed with $5000 \AA$ aluminum using an electron-beam evaporator (Temescal, Wilmington, MA, USA). The wafer was placed in an acetone bath to lift off the photoresist and any excess aluminum, followed by an acetone spray as needed and by the following rinses in methanol, isopropyl alcohol, and deionized water. This process was repeated for ground metallization, bus lines, and contact pads. Next, a waveguide layer of $5000 \AA$ silicon dioxide $\left(\mathrm{SiO}_{2}\right)$ was deposited as a film on the wafer using lift-off plasma enhanced chemical vapor deposition (Oerlikon Versaline, Pfaeffikon, Switzerland). The oxide was coated with hexamethyldisilazane in a vacuum oven, and positive tone photoresist AZ4330 (AZ Electronic Materials) was used to form a photoresist mask on the wafer having exposed portions. The exposed portions of $\mathrm{SiO}_{2}$ was etched with reactive ion etching to access electrical contact pads. Finally, the resultant wafer was diced to form individual chips, in which photoresist from dies was removed by rinsing in acetone, methanol, and isopropanol.

Antibodies were individually patterned on sensing lanes as previously described [16]. Briefly, sensor chips were coated with toluene and 3-glycidyloxypropyl trimethoxysilane (90\%/10\%) in an oven at $60{ }^{\circ} \mathrm{C}$ for $1.5 \mathrm{~h}$ and then rebaked at $100{ }^{\circ} \mathrm{C}$ for $1 \mathrm{~h}$. Each lane was coated with antibodies at a concentration of $10 \mu \mathrm{g} / \mathrm{mL}$ in phosphate-buffered saline (PBS). The following antibodies were used: mouse monoclonal antibodies (IgG2a isotype) specific for Ebola (AB-EB-MAB1, anti-Ebola virus monoclonal antibody 1; BEI Resources, Manassas, VA, USA); and mouse IgG1 antibody isotype control (F(ab')2 fragment) (ab37426, monoclonal isotype control; Abcam, Cambridge, MA, USA). Typically, two lanes were functionalized with antibodies specific for the target analyte, and two lanes were functionalized with isotype control antibodies; the latter are referred to as reference lanes.

\subsection{Provenance and Handling of Ebola Virus Strain Zaire (Mayinga) Inactivated}

The Ebola virus antigen sample (NR-31807; BEI Resources, Manassas, VA, USA) consists of inactivated and highly disrupted viral particles and was used as provided. Fully intact Ebolavirus is highly pathogenic and designated BSL-4 material. For research purposes, the supplier provides inactivated and disrupted Zaire (Mayinga) Ebola virus (BSL-1 material), which was prepared as follows. As stated by the supplier, Zaire (Mayinga) Ebola virus from infected Vero E6 pellets was suspended in $50 \mathrm{mM}$ sodium borate, gamma irradiated $\left(5 \times 10^{6}\right.$ rads total dose $)$ on dry ice, and sonicated. Culture cell debris were removed by centrifugation. The virus was confirmed non-viable (killed or inactivated) by inoculation of cell culture (10 days on Vero cells) followed by a second passage (10 days on Vero cells). The concentration of the Ebola virus antigen sample was provided in plaque-forming units (PFU/mL) by the supplier, indicating the concentration of viable viral particles prior to inactivation, and allowing for comparison across different animal models and experimental conditions [17]. 
The Ebola antigen samples were analyzed by scanning electron microscopy at the University of New Mexico Health Sciences Center Electron Microscopy Facility (Albuquerque, NM, USA) to roughly determine the size distribution of the degraded viral particles. Ebola samples were stored in aliquots of $50 \mu \mathrm{L}$ in screw cap safety microtubes at $-80{ }^{\circ} \mathrm{C}$ until ready for use.

When ready to be tested, the samples were thawed on ice and supplemented with phosphate buffered saline (PBS; $137.0 \mathrm{mM} \mathrm{NaCl}, 2.7 \mathrm{mM} \mathrm{KCl}, 10.0 \mathrm{mM} \mathrm{Na} 2 \mathrm{HPO}_{4} \cdot 2 \mathrm{H}_{2} \mathrm{O}, 2.0 \mathrm{mM} \mathrm{KH} \mathrm{PO}_{4}$, pH 7.4 with $\mathrm{HCl})$. All Ebola antigen samples were prepared fresh for each experiment.

\subsection{Virus Detection Using the $S A W$ Biosensor}

Detection of Ebola Zaire antigen was conducted in BSL-2 certified biosafety cabinets. Data expressed as phase shift ( $\Delta \varphi$, expressed in degrees) were recorded with a custom acquisition program developed using Visual Studio (Microsoft) as previously described [12]. In brief, data from each IDT delay line were collected for each lane and acquired simultaneously as a function of time of acquisition. The biosensor supports a surface shear wave, and the IDT detects the wave by transducing the mechanical wave into an electrical signal. This electrical signal (expressed as voltage information) was converted to phase ( $\varphi$, expressed in degrees) using the custom acquisition program. Data from reference lanes were subtracted from the data from Ebola test lanes at each time point to determine the specific Ebola signal. The data for quantitative measurement of specific Ebola particle detection refer to the phase shift $(\Delta \varphi)$, which corresponds to the difference between the reference and Ebola signals that is measured continuously after addition of a viral antigen sample

Ebola antigen samples of various concentrations were prepared in $100 \mu \mathrm{L}$ of PBS and applied to the biosensor. Binding of viral antigens to the sensor surface resulted in a net phase shift of the signal $\Delta \varphi$ (expressed in degrees on the y-axis) along the time plot (on the x-axis). While phase shift differences associated with specific Ebola signal are apparent and stable within seconds of sample addition, we continue measuring up to $5 \mathrm{~min}$ after sample addition to assure signal stability and reproducibility. All measurements represent multiplicates of at least three, and data points are represented as means \pm standard error of the mean (SEM).

\section{Results and Discussion}

We present here the initial development and characterization of a biosensor for point-of-care, quantitative detection of the Ebola virus. This device enables log-linear, concentration-dependent detection of disrupted viral particles in the clinically relevant range. Overall sensitivity and limit of detection are dependent on the mass of the species that is bound to the sensor surface; this biosensor is likely to be orders of magnitude more sensitive for intact, infectious Ebola viral samples [15].

\subsection{Design Elements of Surface Acoustic Wave Biosensor}

The biosensor was adapted to allow for sensitive detection of the Ebola virus. The biosensor has a planar, piezoelectric substrate containing inter-digital transducers (IDTs) [12,13]. The piezoelectric substrate propagates horizontally polarized surface shear waves, and such waves are induced by applying an alternating voltage to the IDTs at a high frequency (generally between 80 and $400 \mathrm{MHz}$ ). The surface 
shear waves are characterized by a particular resonant frequency that is sensitive to changes on the sensor surface.

Specificity for the target bioagents is obtained by functionalizing the surface of the substrate (Figure 1a). Here, the surface of the piezoelectric substrate was sensitized with a monoclonal antibody specific for Ebola virus. A binding event causes a change in surface mass and results in a phase shift of the signal wave propagating across the sensor surface. Biochemical interactions occurring on the sensor surface can be quantified by measuring this change in phase shift $(\Delta \varphi)$.

The overall schematic concept of the biosensor device for detecting the Ebola virus is shown in Figure 1. The biosensor includes a test lane including antibodies selective for the target bioagents (i.e., the Ebola virus in this study), as well as a reference lane including a control IgG1 antibody (Figure 1a). Measurements of $\Delta \varphi$ were performed for both the test lane and the reference lane by use of an output interface device (connected to the SAW biosensor) and a laptop computer (Figure 1b).

(a)

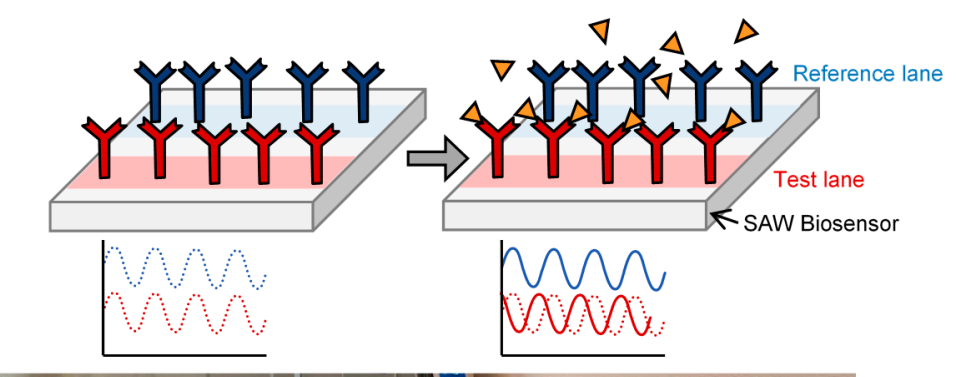

(b)

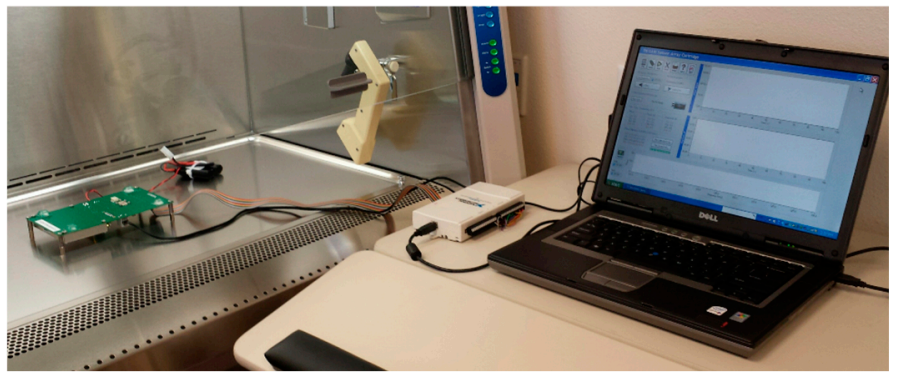

Figure 1. Biosensor. (a) Schematic of a surface acoustic wave (SAW) biosensor having a test lane functionalized with an antibody (red) specific for the target bioagents (triangles). The biosensor also includes a reference lane functionalized with a control antibody (blue); (b) The detection system for research use with fluidic housing surrounding the biosensor, an output interface device connected to the biosensor, and a laptop computer. The sensor is powered by disposable AA, and further miniaturization of the output interface is possible for field use. In addition, the fluidic housing and biosensor can be provided as a disposable module that can be detached from the output interface and then decontaminated prior to disposal.

\subsection{Imaging Analysis of the Ebola Zaire Antigen Sample}

Fully intact Ebolavirus is highly pathogenic and designated BSL-4 material. For this particular study, we employed inactivated and disrupted Zaire (Mayinga strain) Ebola virus (BSL-1 material), which is available for research purposes under non-BSL-4 conditions. Thus, transmission electron microscopy studies were conducted to identify the size distribution of the fragmented viral particles. Representative images are provided in Figure 2. Shown is a TEM image of a 1:20 diluted sample (with PBS), which includes a majority of particles that are less than $10 \mathrm{~nm}$ and the presence of larger aggregates between 
about $50 \mathrm{~nm}$ to $300 \mathrm{~nm}$ (Figure 2a). Rare filamentous structures, with diameter of about $30 \mathrm{~nm}$ (Figure 2b) were also noted. Intact Ebola virus has a filamentous structure with diameter of $80 \mathrm{~nm}$ and length greater than $950 \mathrm{~nm}[18]$.
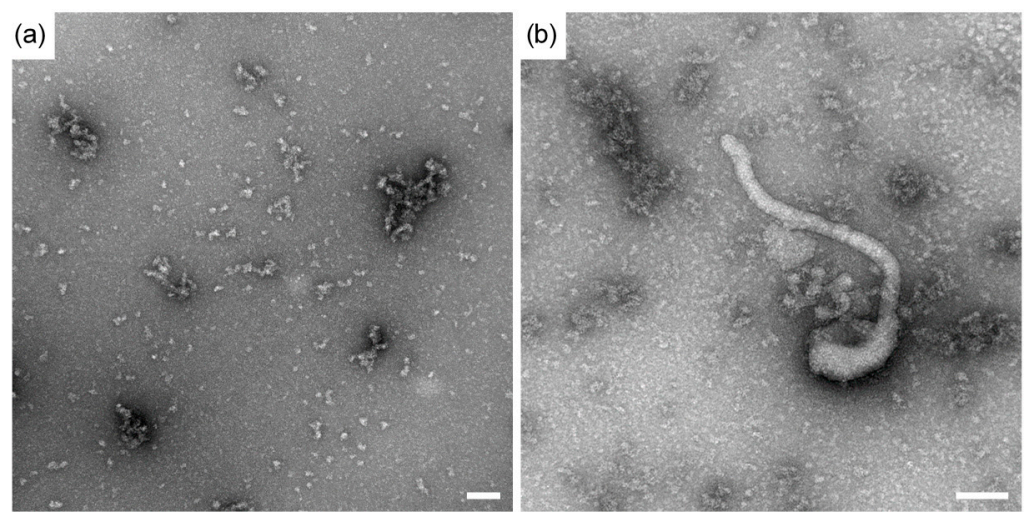

Figure 2. Transmission electron microscopy (TEM) images of inactivated Ebola Zaire virus sample showing predominance of fragmented viral particles. (a) A representative image of dilute samples containing the fragmented, inactivated Ebola virus; (b) An image of rare filamentous structures having a diameter of approximately $30 \mathrm{~nm}$. Scale bars are $100 \mathrm{~nm}$.

TEM of the inactivated virus sample shows highly disrupted, fragmented particles that form aggregates (Figure 2a), rather than the elongated filaments observed in intact filovirus. When filamentous structures were observed (Figure 2b), the cross-section diameter was less than half of the diameter expected for intact filovirus [18]. While the predominant species in the inactivated virus sample are disrupted particles, the biosensor phase shift response is likely to be much greater for an infectious Ebola sample containing intact virus. Based on our previous study of HIV detection [16] and the relative masses of intact HIV and Ebola $[18,19]$, we would estimate a 5-10× greater sensitivity for intact Ebola virus compared to intact HIV. As a first approximation, we assume a log-linear correlation between mass and phase shift. As particles of greater mass will provide a greater response, the sensor is likely to detect more massive, intact viral particles at lower concentration.

\subsection{SAW Biosensor Detection of the Ebola Zaire Sample}

In analogy to our previous report on the detection of HIV-1 and HIV-2 [16], we functionalized the SAW biosensor with monoclonal antibodies specific for Zaire (Mayinga) strain of Ebola Virus. Ebola virus antigens were detected in PBS solutions over a $2.5 \mathrm{log}$ reference range at concentrations corresponding to $1.0 \times 10^{4} \mathrm{PFU} / \mathrm{mL}$ to $3.0 \times 10^{6} \mathrm{PFU} / \mathrm{mL}$ prior to virus inactivation. The lowest concentration is below the average viremia level of $3 \times 10^{4}$ RNA copies per mL observed in Ebola patients on the first day of disease symptoms [20].

Representative phase shift data are provided in Figure 3. The IgG control lane accounts for matrix effects and non-specific binding of potentially interfering species.

Detection of Ebola virus resulted in a concentration-dependent increase with $\Delta \varphi$ values ranging from $0.20 \pm 0.04$ to $4.46 \pm 0.86$, corresponding approximately to $1.6 \times 10^{4} \mathrm{PFU} / \mathrm{mL}$ to $6.5 \times 10^{6} \mathrm{PFU} / \mathrm{mL}$ (Figure 4). There was a log-linear relationship between viral load and $\Delta \varphi$ for this concentration range of viral particles with a correlation coefficient $R^{2}$ of 0.92 . The linear range and correlation coefficients compare 
favorably to those recently reported for qRT-PCR [21]. A limit of detection (LOD) of $1.9 \times 10^{4} \mathrm{PFU} / \mathrm{mL}$ was calculated for Ebola virus by linear regression and using the average background noise $\Delta \varphi$ value of 0.31 . These results indicate that the prototype SAW biosensor rapidly detects the Zaire strain of Ebola antigens in a defined buffer with detection limits below the average viremia level at onset of clinical symptoms.

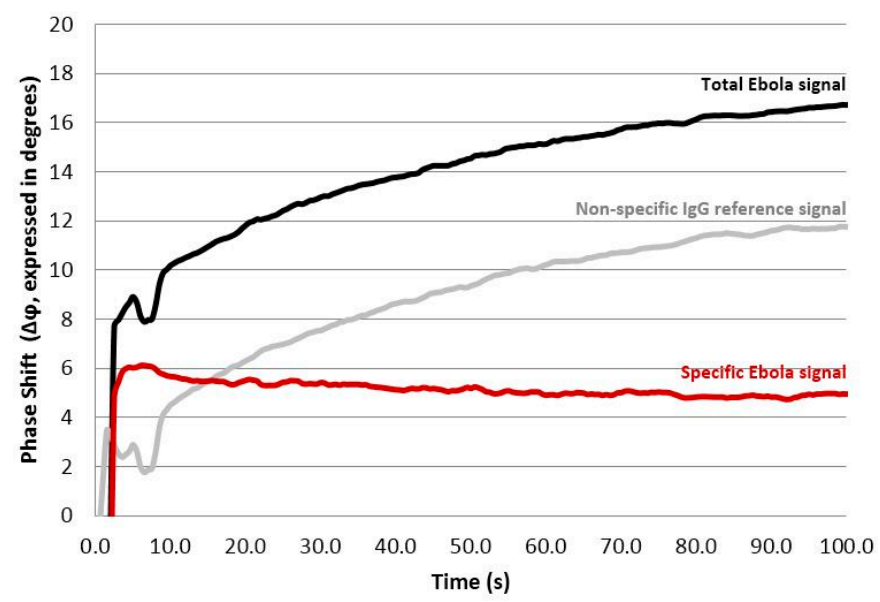

Figure 3. Sensor response and calculated phase shift $\Delta \varphi$ in degrees as a function of acquisition time. Graph showing data from Ebola (solid black line) and reference (gray line) IDTs for test viral antigen with a concentration of 1,000,000 PFU $/ \mathrm{mL}$. The specific Ebola signal (calculated by subtracting the Non-specific IgG signal from the Total Ebola signal) is also shown (red line). Sample addition occurs at time 0 , and several seconds are required for the signal to stabilize.

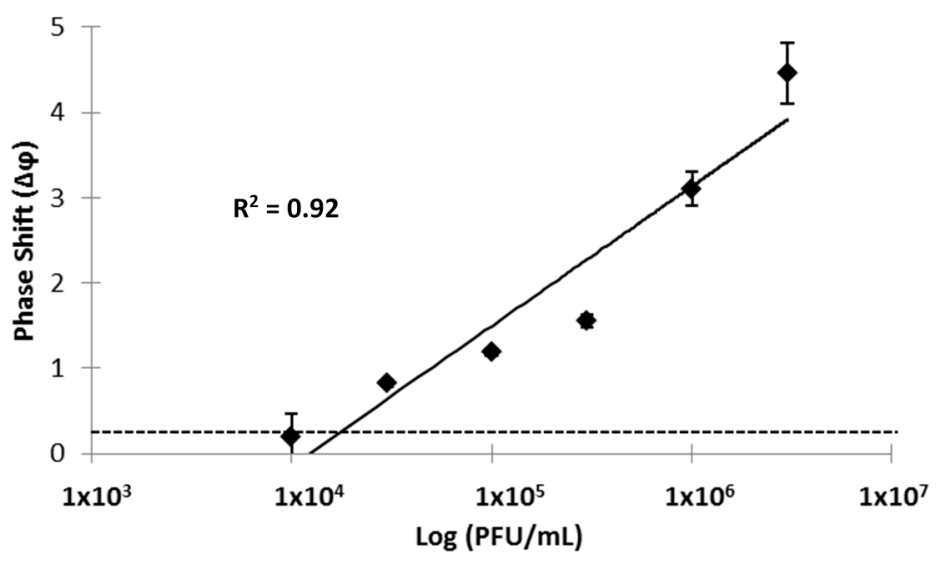

Figure 4. Detection of Ebola-Zaire with a SAW biosensor functionalized with an anti-Ebola monoclonal antibody. Triplicate measurements (mean \pm standard deviation) were taken at 5 min after addition of the virus. Standard deviations for three of the data points are smaller than the corresponding size of the data marker. The x-axis and y-axis indicate viral load (logarithmic scale; PFU/mL) and phase shifts $(\Delta \varphi$, expressed in degrees), respectively. Concentrations of Ebola antigen corresponding to PFU of intact virus were established in a defined buffer (PBS). The solid line represents a linear fit and the correlation coefficient $\left(R^{2}\right)$ of 0.92 suggests operation in a linear range. The horizontal dotted line represents the background signal obtained with buffer without virus, and is the basis for LOD calculations. 


\section{Conclusions/Outlook}

We have demonstrated an adaptable, label-free sensing system for the rapid detection of bioagents and show its use to detect the Ebola Zaire virus. We have previously shown the effectiveness of this system in detecting the Bacillus anthracis bacteria simulant, Bacillus thuringiensis [13]; the Coxsackie virus [12]; the Sin Nombre hantavirus [12]; the Francisella tularensis bacteria [22] and the Human Immunodeficiency Virus types 1 and 2 [16]. This study further extends the capability of this SAW biosensor platform to accommodate the rapid, label-free, and specific detection of the Ebola Zaire virus.

As fully intact Ebola virus is highly contagious, we used an inactivated virus in order to conduct this study under non-BSL-4 conditions. However, in a first responder or real world scenario, the sample would not necessarily have to be inactivated and could be tested directly, providing the sensor was contained in a controlled environment.

The SAW biosensor described here provides the basis for a rapid and specific response to Ebola outbreaks and other emerging diseases. This point-of-care sensor will provide rapid diagnosis and improved infection control, dramatically decreasing the human and economic costs of this disease. Further work will focus on portability and optimization for field use. A disposable cartridge can be employed to house the piezoelectric substrate and fluidic channels to guide the test sample to the substrate. The user interface can be readily simplified to provide a simple positive/negative result to a field worker on the front lines of the next epidemic.

Sample preparation is not required for this label-free sensing methodology, simplifying use in field conditions without centralized laboratories or refrigeration. While additional testing can be conducted to verify the detection limits for Ebola Zaire in complex media, such as blood, serum, or saliva [23], we believe that Ebola virus detection in such media will be guided by our past studies that show effective detection of other viruses in complex solutions (e.g., serum, plasma, river water, and sewage effluent $[12,14,16]$ ). In summary, the SAW biosensor is a versatile platform that shows promise to revolutionize rapid pathogen detection and enable early treatment in public health and emergency responses.

\section{Acknowledgments}

We thank Stephen Jett of the UNM Electron Microscopy Shared Facility for assistance with TEM. The following reagent was obtained through BEI Resources, NIAID, NIH: Zaire Ebolavirus, Mayinga, Gamma-Irradiated, NR-31807. The following reagent was obtained from the Department of Defense Critical Reagents Program (CRP) via BEI Resources, DD-24 Ebolavirus, AB-EB-MAB1 anti-Ebola virus monoclonal antibody 1. We acknowledge support from NIH-NCATS grant 8KL2TR000089-03; Clinical and Translational Science Award, University of New Mexico.

\section{Author Contributions}

J.T.B. and R.S.L. designed the experiments; D.W.B. fabricated biosensor materials; V.S., J.T.B., and D.L. performed the experimental work; J.T.B., V.S., and D.L. drafted the manuscript, all authors reviewed and accepted the final version of the manuscript; and R.S.L oversaw the project. 


\section{Conflicts of Interest}

The authors declare no conflict of interest.

\section{References}

1. Frieden, T.R.; Damon, I.; Bell, B.P.; Kenyon, T.; Nichol, S. Ebola 2014-New challenges, new global response and responsibility. N. Engl. J. Med. 2014, 371, 1177-1180.

2. Hampton, T. Largest-ever outbreak of Ebola Virus disease thrusts experimental therapies, vaccines into spotlight. JAMA 2014, 312, 987-989.

3. World Health Organization, What This - The largest Ebola Outbreak in History_-Tells the World. Available online: http://www.who.int/csr/disease/ebola/ebola-6-months/lessons/en/ (accessed on 23 March 2015).

4. Centers for Disease Control and Prevention, Ebola Outbreaks 2000-2014. Available online: http://www.cdc.gov/vhf/ebola/outbreaks/history/summaries.html (accessed on 23 March 2015).

5. World Health Organization, Urgently Needed: Rapid, Sensitive, Safe and Simple Ebola Diagnostic Tests. Available online: http://www.who.int/mediacentre/news/ebola/18-november2014-diagnostics/en/ (accessed on 23 March 2015).

6. Chertow, D.S.; Kleine, C.; Edwards, J.K.; Scaini, R.; Giuliani, R.; Sprecher, A. Ebola Virus Disease in West Africa-Clinical manifestations and management. N. Engl. J. Med. 2014, 371, 2054-2057.

7. MacNeil, A.; Rollin, P.E. Ebola and Marburg hemorrhagic fevers: Neglected tropical diseases? PLoS Negl. Trop. Dis. 2012, 6, e1546.

8. Tseng, C.P.; Chan, Y.J. Overview of Ebola virus disease in 2014. J. Chin. Med. Assoc. 2015, 78, $51-55$.

9. WHO Ebola Response Team. Ebola virus disease in West Africa-The first 9 months of the epidemic and forward projections. N. Engl. J. Med. 2014, 16, 1481-1495.

10. Bartsch, S.M., Gorham, K.; Lee, B.Y. The cost of an Ebola case. Pathog. Glob. Health 2015, 109, 4-9.

11. Schieffelin, J.S.; Shaffer, J.G.; Goba, A.; Gbakie, M.; Gire, S.K.; Colubri, A.; Sealfon, R.S.; Kanneh, L; Moigboi, A.; Momoh, M.; et al. Clinical illness and outcomes in patients with Ebola in Sierra Leone. N. Engl. J. Med. 2014, 371, 2092-2100.

12. Bisoffi, M.; Hjelle, B.; Brown, D.C.; Branch, D.W.; Edwards, T.L.; Brozik, S.M.; Bondu-Hawkins, V.S.; Larson, R.S. Detection of viral bioagents using a shear horizontal surface acoustic wave biosensor. Biosens. Bioelectron. 2008, 23, 1397-1403.

13. Branch, D.W.; Brozik, S.M. Low-level detection of a bacillus anthracis simulant using Love-wave biosensors on 36 degrees YX LiTaO3. Biosens. Bioelectron. 2004, 19, 849-859.

14. Larson, R.S.; Baca, J.T. Versatility of the surface acoustic wave biosensor platform for detecting bacteria and viruses. In Global Point of Care: Strategies for Disasters, Emergencies, and Public Health Resilience; Kost, G.J., Curtis, C.M., Eds.; AACC Press: Washington, DC, USA, 2015; pp. 165-172.

15. Gronewold, T.M. Surface acoustic wave sensors in the bioanalytical field: recent trends and challenges. Anal. Chim. Acta 2007, 603, 119-128. 
16. Bisoffi, M.; Severns, V.; Branch, D.W.; Edwards, T.L.; Larson, R.L. Rapid detection of human immunodeficiency virus types 1 and 2 by use of an improved piezoelectric biosensor. J. Clin. Microbiol. 2013, 51, 1685-1691.

17. Bente, D., Gren, J.; Strong, J.E.; Feldmann, H. Disease modeling for Ebola and Marburg viruses. Dis. Model. Mech. 2009, 2, 12-17.

18. Geisbert, T.W.; Jahrling, P.B. Use of immunoelectron microscopy to show Ebola virus during the 1989 United States epizootic. J. Clin. Pathol. 1990, 43, 813-816.

19. Gentile, M.; Adrian, T.; Scheidler, A.; Ewald, M.; Dianzani, F.; Pauli, G.; Gelderblom, H.R. Determination of the size of HIV using adenovirus type 2 as an internal length marker. J. Virol. Methods 1994, 48, 43-52.

20. Towner, J.S.; Rollin, P.E.; Bausch, D.G.; Sanchez, A.; Crary, S.M.; Vincent, M.; Lee, W.F.; Spiropoulou, C.F.; Ksiazek, T.G.; Lukwiya, M.; et al. Rapid diagnosis of Ebola hemorrhagic fever by reverse transcription-PCR in an outbreak setting and assessment of patient viral load as a predictor of outcome. J. Virol. 2004, 78, 4330-4341.

21. Rossi, C.A.; Kearney, B.J.; Olschner, S.P.; Williams, P.L.; Robinson, C.G.; Heinrich, M.L.; Zovanyi, A.M.; Ingram, M.F.; Norwood, D.A.; Schoepp, R.J. Evaluation of ViroCyt ${ }^{\circledR}$ Virus Counter for Rapid Filovirus Quantitation. Viruses 2015, 7, 857-872.

22. Bisoffi, M.; Severns, V.; Larson, R. CTSA-enhanced innovative device development. Clin. Transl. Sci. 2012, 5, 311-313.

23. Beeching, N.J.; Fenech, M.; Houlihan, C.F. Ebola virus disease. BMJ 2014, 349, g7348. Available online: http://www.bmj.com/content/349/bmj.g7348 (accessed on 23 March 2015).

(C) 2015 by the authors; licensee MDPI, Basel, Switzerland. This article is an open access article distributed under the terms and conditions of the Creative Commons Attribution license (http://creativecommons.org/licenses/by/4.0/). 